\section{Phosphorylation of H4 Ser 47 promotes HIRA-mediated nucleosome assembly}

\author{
Bin Kang, ${ }^{1,5}$ Mintie Pu, ${ }^{1,5}$ Gangqing $\mathrm{Hu}^{2}$ \\ Weihong Wen, ${ }^{3}$ Zigang Dong, ${ }^{3}$ Keji Zhao, ${ }^{2}$ \\ Bruce Stillman, ${ }^{4}$ and Zhiguo Zhang ${ }^{1,6}$
}

${ }^{1}$ Department of Biochemistry and Molecular Biology, Mayo Clinic College of Medicine, Rochester, Minnesota 55905, USA; ${ }^{2}$ Laboratory of Molecular Immunology, National Heart, Lung, and Blood Institute, National Institutes of Health, Bethesda, Maryland 20892, USA; ${ }^{3}$ The Hormel Institute, University of Minnesota, Austin, Minnesota 55912, USA; ${ }^{4}$ Cold Spring Harbor Laboratory, Cold Spring Harbor, New York 11724, USA

Histone $\mathrm{H} 3$ variant $\mathrm{H} 3.3$, while differing from canonical H3 (H3.1) by only five amino acids, is assembled into nucleosomes, along with histone $\mathrm{H} 4$, at genic regions by the histone chaperone HIRA, whereas H3.1 is assembled into nucleosomes in a CAF-1-dependent reaction. Here, we show that phosphorylation of histone H4 Ser 47 (H4S47ph), catalyzed by the PAK2 kinase, promotes nucleosome assembly of $\mathrm{H} 3.3-\mathrm{H} 4$ and inhibits nucleosome assembly of H3.1-H4 by increasing the binding affinity of HIRA to $\mathrm{H} 3.3-\mathrm{H} 4$ and reducing association of CAF-1 with $\mathrm{H} 3.1-\mathrm{H} 4$. These results reveal a mechanism whereby $\mathrm{H} 4 \mathrm{~S} 47 \mathrm{ph}$ distinctly regulates nucleosome assembly of $\mathrm{H3} .1$ and $\mathrm{H} 3.3$.

Supplemental material is available for this article.

Received March 31, 2011; revised version accepted May 25, 2011.

The basic repeating unit of chromatin is the nucleosome, consisting of 146 base pairs of DNA wrapped around a histone octamer containing one $(\mathrm{H} 3-\mathrm{H} 4)_{2}$ tetramer and two H2A-H2B dimers. To form a nucleosome, $(\mathrm{H} 3-\mathrm{H} 4)_{2}$ tetramers are deposited first, followed by rapid deposition of two H2A-H2B dimers. Thus, assembly of $(\mathrm{H} 3-\mathrm{H} 4)_{2}$ tetramers into nucleosomes is believed to be a key step in chromatin formation following DNA replication, gene transcription, and DNA repair (Groth et al. 2007; Morrison and Shen 2009; Ransom et al. 2010; Szenker et al. 2011). Furthermore, deregulation of $\mathrm{H} 3-\mathrm{H} 4$ nucleosome assembly is linked to aging and cancer development (Das et al. 2009; Ransom et al. 2010). Thus, it is important to understand how nucleosome assembly of $\mathrm{H} 3-\mathrm{H} 4$ is regulated.

In addition to the canonical histone $\mathrm{H} 3$ (H3.1), chromatin in higher eukaryotic cells also contains the histone H3 variant H3.3 (Elsaesser et al. 2010; Szenker et al. 2011). While it differs from the canonical histone H3.1 by only five amino acids, H3.3 has unique functions. For instance, Drosophila cells lacking both copies of H3.3

[Keywords: CAF-1; HIRA; nucleosome assembly; PAK2; histone H3.3; histone variant]

${ }^{5}$ These authors contributed equally to this work.

${ }^{6}$ Corresponding author.

E-mail Zhang.Zhiguo@mayo.edu.

Article is online at http://www.genesdev.org/cgi/doi/10.1101/gad.2055511. exhibit widespread transcriptional defects and male sterility (Sakai et al. 2009). In mice, mutations in one of the H3.3 genes result in postnatal death in 50\% of homozygous mutants and male infertility (Couldrey et al. 1999). Thus, the function of $\mathrm{H} 3.3$ cannot be substituted by H3.1. Early studies indicated that H3.3 was enriched at gene bodies of actively transcribed genes, and the levels of H3.3 at gene bodies positively correlated with gene expression (Mito et al. 2005; Schwartz and Ahmad 2005). H3.3 also has a role in heterochromatin formation and gene silencing. For instance, H3.3, but not H3.1, plays an important role in heterochromatin formation during mouse development (Santenard et al. 2010). Using chromatin immunoprecipitation and sequencing (ChIP-seq) technology, H3.3 has been found at the promoters of both active and inactive genes in human cells (Jin et al. 2009) and mouse embryonic fibroblasts (MEFs) (Goldberg et al. 2010). Thus, H3.3 appears to regulate both active chromatin and heterochromatin.

H3.1 and H3.3 are assembled into nucleosomes via distinct nucleosome assembly pathways. $\mathrm{H} 3.1-\mathrm{H} 4$ is assembled into nucleosomes by chromatin assembly factor 1 (CAF-1) in a DNA replication-coupled (RC) nucleosome assembly process (Smith and Stillman 1989; Tagami et al. 2004). In contrast, two distinct proteins have been described for nucleosome assembly of $\mathrm{H} 3.3-\mathrm{H} 4$ molecules, which occurs primarily via a DNA replication-independent (RI) process. HIRA was the first histone $\mathrm{H} 3.3-\mathrm{H} 4$ chaperone found to promote nucleosome assembly of $\mathrm{H} 3.3-\mathrm{H} 4$ in mammalian cells (Ray-Gallet et al. 2002; Tagami et al. 2004). Recently, the death domain-containing protein (Daxx), which forms a complex with the chromatin remodeling protein ATRX, has been identified as another H3.3-H4 chaperone (Drane et al. 2010; Goldberg et al. 2010). It has been shown that the H3.3 localization at genic regions, including both active and inactive genes, depends on HIRA, whereas the telomeric localization of $\mathrm{H} 3.3$ depends on ATRX in MEFs (Goldberg et al. 2010). Thus, it appears that the localization of $\mathrm{H} 3.3$ at distinct chromatin domains depends on different histone chaperones, and understanding how nucleosome assembly of H3.3 is regulated will shed light on the function of H3.3 at distinct chromatin domains.

Acetylation of newly synthesized $\mathrm{H} 3$ plays an important role in the regulation of RC nucleosome assembly in Saccharomyces cerevisiae (Chen et al. 2008; Li et al. 2008; Burgess et al. 2010). However, how modifications on newly synthesized H3 and H4 impact nucleosome assembly in mammalian cells has not been well studied. Here, we show that phosphorylation of histone H4 Ser 47 (H4S47ph), a modification whose function has not been characterized previously, is catalyzed by the PAK2 kinase and is enriched in H3.3-containing nucleosomes. In addition, we provide evidence in vitro and in vivo to support the model that H4S47ph promotes HIRA-mediated nucleosome assembly and inhibits CAF-1-mediated nucleosome assembly by regulating the interactions of HIRA and CAF1 with their corresponding $\mathrm{H} 3-\mathrm{H} 4$ molecules.

\section{Results and Discussion}

Histone H4 associated with Asf1a and Asf1b is phosphorylated at Ser 47

Asfla and Asf1b are two mammalian homologs of yeast Asf1 that bind newly synthesized histones and function 
in both RC and RI nucleosome assembly (Ransom et al. 2010). To identify modifications on newly synthesized H3 and $\mathrm{H} 4$ in mammalian cells, we purified Asfla- and Asflbcontaining protein complexes from 293T S100 cytosol cell extracts and used mass spectrometry to identify Asfla- and Asflb-associated proteins and modifications on the associated histone $\mathrm{H} 3$ and $\mathrm{H} 4$. The majority of proteins that copurified with Asfla and Asf1b were similar to what has been reported (Supplemental Fig. S1A-C; Jasencakova et al. 2010). In addition to the histone acetylation that has been reported (Jasencakova et al. 2010), we also found that histone $\mathrm{H} 4$ copurifying with Asfla and Asflb was phosphorylated at Ser 47 (H4S47ph) (Supplemental Fig. S1D). While H4S47ph was reported (Benner et al. 1995), the function of this modification was not known. Therefore, we decided to characterize this modification.

First, an antibody against an $\mathrm{H} 4$ peptide phosphorylated at Ser 47 was generated. This antibody recognized endogenous $\mathrm{H} 4$ in $293 \mathrm{~T}$ cell extracts, but neither recombinant H4 purified from Escherichia coli (Fig. 1A, top panel) nor H4 in 293 T cell extracts treated with phosphatase (Supplemental Fig. S2A). In addition, it recognized exogenously expressed $\mathrm{H} 4$ tagged with both Flag and HA epitopes (e-H4), but not the corresponding H4 mutant with Ser 47 mutated to glutamate (e-H4S47E) (Fig. 1A, bottom panel). These results demonstrate that the H4S47ph antibody is specific. Using this antibody, we confirmed via Western blot that H4 copurifying with both Asfla and Asflb was phosphorylated at H4S47 (Fig. 1B). Thus, in addition to acetylation, histone $\mathrm{H} 4$ associated with Asfla and Asflb is also phosphorylated at Ser 47.

\section{PAK2 phosphorylates nonnucleosomal H4S47 in vitro and in vivo}

Previous reports show that the p21-activated protein kinase PAK2, best known for its role in cytoskeletal remodeling (Benner et al. 1995; Bokoch 2003), can phosphorylate an H4S47-containing peptide in vitro (Benner et al. 1995); however, the specificity of PAK2 toward histone H4S47 was not tested. To address this, wild-type PAK2 and a kinase-dead (KD) mutant (K278R) were purified and tested for their ability to phosphorylate four different substrates in vitro: a H4S47 peptide, mononucleosomes (MN), core histones (CO), and recombinant $\mathrm{H} 3-$ $\mathrm{H} 4$ tetramers. PAK2, but not the KD mutant, phosphorylated the H4S47 peptide (Supplemental Fig. S2B). Moreover, PAK2 exhibited robust activity toward $\mathrm{H} 4$ and $\mathrm{H} 4 \mathrm{~S} 47$ in core histones and $\mathrm{H} 3-\mathrm{H} 4$ tetramers, but little, if any, activity toward mononucleosomes (Fig. 1C; Supplemental Fig. S2C). Furthermore, PAK2 had no detectable activity toward mutant $\mathrm{H} 3-\mathrm{H} 4$ tetramers containing a mutation at H4S47 (S47A), but exhibited activity toward mutant H3$\mathrm{H} 4$ tetramers with a mutation at $\mathrm{H} 4 \mathrm{Thr} 80$ (T80A) (Fig. 1D,E). Both S47 and T80 of H4 are considered to be PAK2 phosphorylation consensus sites (Bokoch 2003). Finally, PAK2 exhibited limited activity against $\mathrm{H} 3$ in vitro, and this activity depended on H4S47 phosphorylation (Fig. 1D). Together, these experiments demonstrate that PAK2 purified from mammalian cells preferentially phosphorylates nonnucleosomal H4S47 in vitro.

To determine whether H4S47 phosphorylation depends on PAK2 in vivo, we depleted PAK2 in HeLa cells using different shRNAs. The level of H4S47ph was significantly reduced in PAK2-depleted cells as detected by both immunofluorescence and Western blot (Fig. 1F; Supplemental

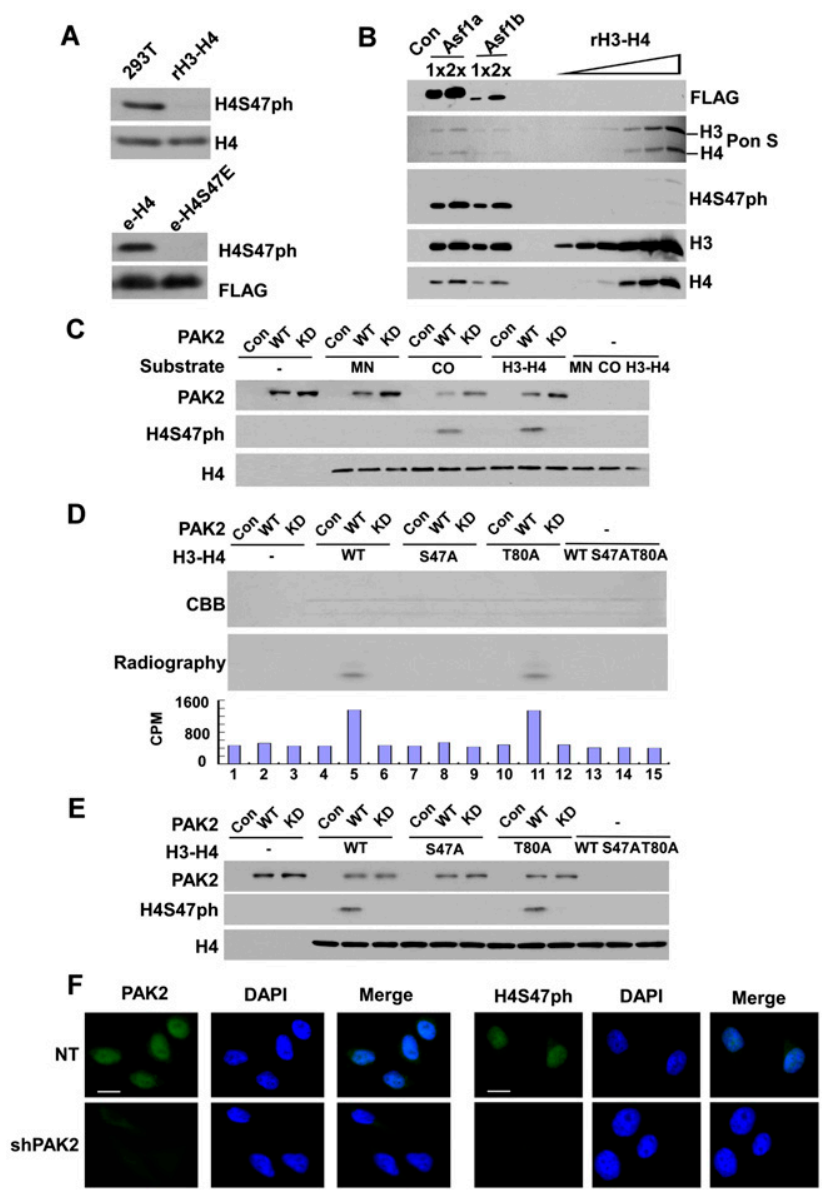

Figure 1. PAK2 phosphorylates nonnucleosomal H4S47 in vitro and in vivo. (A) The antibody generated against $\mathrm{H} 4 \mathrm{~S} 47 \mathrm{ph}$ is specific. Cell lysates from $293 \mathrm{~T}$ cells and recombinant $\mathrm{H} 3-\mathrm{H} 4$ (rH3-H4) (shown in the top panel), and immunoprecipitation samples of exogenously expressing $\mathrm{H} 4$ tagged with the Flag and HA epitopes (e-H4), or the corresponding H4 mutant with S47 mutated to E (e-H4S47E) were analyzed by Western blot using an antibody prepared against a $\mathrm{H} 4 \mathrm{~S} 47 \mathrm{ph}$ peptide. $(B) \mathrm{H} 4$ associated with Asfla and Asf1b is phosphorylated at S47 by PAK2. Asfla and Asf1b were purified from 293 T cells, and copurified histones (left) and different amounts of rH3-H4 (right) were analyzed by Western blot using antibodies against $\mathrm{H} 4 \mathrm{~S} 47 \mathrm{ph}, \mathrm{H} 3$, or $\mathrm{H} 4$. The membrane was stained with Ponceau S (Pon S) before proceeding for Western blot. (C) PAK2 phosphorylates H4S47 of nonnucleosomal H4 in vitro. PAK2 was purified from $293 \mathrm{~T}$ cells and then incubated with ATP and different histone substrates, including mononucleosomes (MN), core histones (CO), and rH3-H4. As controls, a kinase-dead (KD) PAK2 mutant (K278R) and a vector control (Con) were transfected into $293 \mathrm{~T}$ cells and purified using the same procedure as the PAK2 purification. The reaction mixtures were analyzed by Western blot using antibodies against PAK2, H4S47ph, and H4. $(D, E)$ PAK2 predominately phosphorylates H4S47 in vitro. The experiments were performed as described in $\mathrm{C}$ using recombinant $(\mathrm{H} 3-\mathrm{H} 4)_{2}$ tetramers and two mutant $(\mathrm{H} 3-\mathrm{H} 4)_{2}$ tetramers with $\mathrm{S} 47$ or $\mathrm{T} 80$ of $\mathrm{H} 4$ changed to alanine (S47A and T80A) and $\left[\gamma^{32} \mathrm{P}\right]$-ATP $(D)$ or ATP $(E)$. For kinase assays using $\left[\gamma_{-}{ }^{32} \mathrm{P}\right]$-ATP, half of the reaction mixtures were resolved on SDS-PAGE. Proteins were detected by Coomassie Brilliant Blue (CBB) staining, and phospho-proteins were detected by antoradiography. $(F)$ PAK2 phosphorylates H4S47 in vivo. HeLa cells were infected with virus targeting PAK2 (shPAK2) or a nontargeting control (NT). Cells were stained with 4',6-diamidino-2-phenylindole (DAPI) and antibodies against PAK2 or H4S47ph for detection by a fluorescence microscope. Bar, $10 \mu \mathrm{m}$. 
Fig. S2D). These results suggest that PAK2 phosphorylates H4S47 in vivo. Supporting this idea, PAK2, but not the PAK2 KD mutant, bound to histones $\mathrm{H} 3$ and $\mathrm{H} 4$ in vivo (Supplemental Fig. S2E). Thus, H4S47ph depends on PAK2 in vivo.

\section{H4S47ph promotes assembly of H3.3-H4 into nucleosomes}

$\mathrm{H} 4 \mathrm{~S} 47 \mathrm{ph}$ could be detected on $\mathrm{H} 4$ from soluble and chromatin fractions (see Fig. 4, below). To determine where H4S47ph is enriched on chromatin, we first asked whether H4S47ph preferentially associates with $\mathrm{H} 3.1$ or H3.3 nucleosomes. To do this, e-H3.1- and e-H3.3-containing mononucleosomes were isolated using affinity chromatography from HeLa cells stably expressing exogenous H3.1 (e-H3.1) or H3.3 (e-H3.3), each tagged with both the Flag and HA epitopes (Supplemental Fig. S3A), and the levels of H4S47ph on H4 were analyzed by Western blot. H4S47ph levels in e-H3.3 containing mononucleosomes were higher than those in e-H3.1-containing mononucleosomes (Fig. 2A). In contrast, H4S47ph levels on H4 in complex with soluble e-H3.1 and e-H3.3 were similar (Supplemental Fig. $\mathrm{S} 3 \mathrm{~B})$, suggesting that $\mathrm{H} 4 \mathrm{~S} 47 \mathrm{ph}$ preferentially promotes assembly of $\mathrm{H} 3.3-\mathrm{H} 4$ into nucleosomes. To test this idea further, we used ChIP to determine whether H4S47ph is present at H3.3-enriched genes (Jin et al. 2009). In both e-H3.3 and e-H3.1 cell lines, H4S47ph was enriched at three H3.3-enriched genes tested, including the transcription start sites (TSS) of OSTF1 and TP53TG1 and the transcription termination site (TTS) of TM4SF1, compared with TRIM42 and CSRP3, two H3.1-enriched genes (Fig. 2B; Jin et al. 2009).

If H4S47ph promotes assembly of H3.3$\mathrm{H} 4$ into nucleosomes, one would predict that altered levels of H4S47ph would affect H3.3 and H3.1 occupancy on chromatin. Indeed, using ChIP assays, we observed that depleting PAK2 resulted in a significant reduction in $\mathrm{H} 4 \mathrm{~S} 47 \mathrm{ph}$ (Fig. 2C) and e-H3.3 occupancy (Fig. 2D) at the three H3.3enriched genes tested (TM4SF1, OSTF1, and TP53G1) compared with control shRNA-treated cells. In contrast, the e-H3.1 occupancy increased in PAK2-depleted cells at all five genes tested (Fig. 2E), which was probably due to compensation for the loss of H3.3 occupancy resulting from PAK2 depletion. Thus, depletion of PAK2 negatively affects H3.3 occupancy.

In addition to H4S47, PAK2 also phosphorylates $\mathrm{c}-\mathrm{Myc}$, and this phosphorylation negatively regulates the transcriptional activity of Myc (Huang et al. 2004). Therefore, we examined how expression of the phosphomimic H4S47E mutant, c-Myc, or a c-Myc mutant with the PAK2 phosphorylation sites mutated to alanine affects H3.3 occupancy using ChIP assays. Expression of H4S47E increased the H3.3 occupancy at all five candidate genes tested (Fig. 2F). In contrast, cells expressing Myc or the Myc mutant had no apparent effect on H3.3
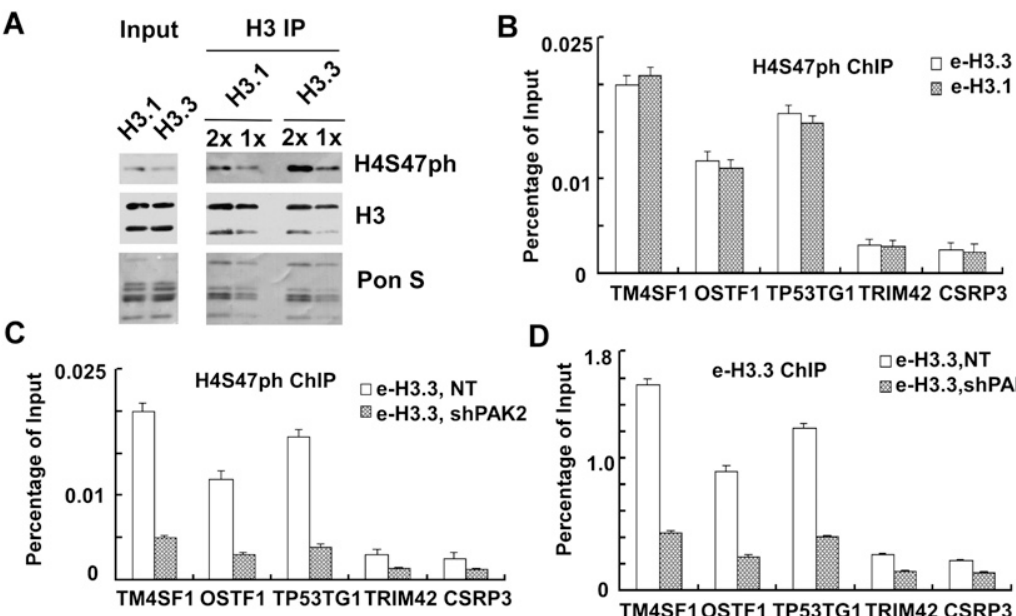

D
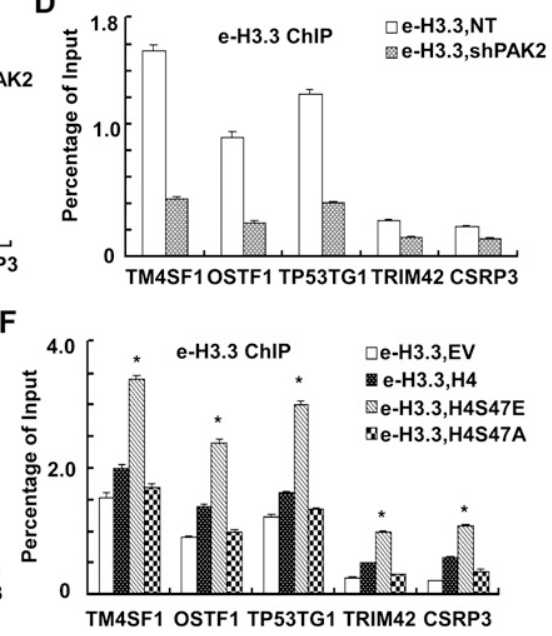

Figure 2. H4S47ph promotes formation of $\mathrm{H} 3.3-\mathrm{H} 4$ nucleosomes. (A) H4S47ph was $\mathrm{H} 4 \mathrm{~S} 47 \mathrm{ph}$ is enriched at three $\mathrm{H} 3.3$-enriched genes tested. ChIP assays were performed in to three H3.3-enriched genes (the TTS of TM4SF1 and the TSS of OSTF1 or TP53G1) and occupancy at candidate genes tested. The e-H3.3 and e-H3.1 cell lines were infected with virus containing shRNA for PAK2 (shPAK2) or a nontargeting (NT) vector, and genes. Empty vector (EV), e-H4, e-H4S47E, or e-H4S47A mutant was expressed in the e-H3.3 cell line. ChIP assays were performed using antibodies against e-H3.3, and coprecipitated DNA was analyzed using real-time PCR. S3C,D). These results strongly suggest that the impact of PAK2 depletion on $\mathrm{H} 3.3$ occupancy at candidate genes is mediated by $\mathrm{H} 4 \mathrm{~S} 47 \mathrm{ph}$ and provide further support for the idea that H4S47ph promotes $\mathrm{H} 3.3-\mathrm{H} 4$ nucleosome assembly.

\section{H4S47ph increases the association of H3.3-H4 with HIRA}

To determine how H4S47ph affects nucleosome assembly of $\mathrm{H} 3.3-\mathrm{H} 4$, we first examined whether $\mathrm{H} 4 \mathrm{~S} 47 \mathrm{ph}$ affects the ability of $\mathrm{H} 4$ to bind $\mathrm{H} 3.1$ and H3.3. Using the phospho-mimic H4S47E mutant, we found that H4S47E bound to H3.1 and H3.3 equally well (Supplemental Fig. S4A). Moreover, PAK2 exhibited similar activity toward H3.1-H4 and H3.3-H4 with or without Asf1a or Asf1b in vitro (Supplemental Fig. S4B,C) and bound e-H3.1 and e-H3.3 equally well (Supplemental Fig. S4D). These results 
suggest that PAK2 and H4S47ph are unlikely to distinguish between $\mathrm{H} 3.1$ and H3.3.

Next, we asked whether alterations in the levels of H4S47ph affect the amount of H3-H4 that copurifies with the corresponding histone chaperones of H3.1 and H3.3. Substantially less CAF-1 (as detected by both the p150 and p60 subunits) and Daxx, but significantly more HIRA, copurified with e-H4S47E than with e-H4 (Fig. 3A,B), whereas similar amounts of Asf1 copurified with both e-H4 and e-H4S47E (Fig. 3A). These results indicate that $\mathrm{H} 4 \mathrm{~S} 47 \mathrm{ph}$ increases the association of H3.3-H4 with HIRA and inhibits the association of $\mathrm{H} 3-\mathrm{H} 4$ with both CAF1 and Daxx. Consistent with this idea, more HIRA and less CAF-1 copurified with e-H4 from cells treated with okadaic acid (OA) than untreated cells (Supplemental Fig. S4E). OA is an inhibitor of protein phosphatases, and OA treatment resulted in a significant increase in $\mathrm{H} 4 \mathrm{~S} 47 \mathrm{ph}$ (Supplemental Fig. S4D). In contrast, the amount of $\mathrm{H} 3-\mathrm{H} 4$ that copurified with HIRA was substantially reduced in PAK2-depleted cells compared with control cells (Fig. 3C, cf. lane 11 and 12), whereas significantly more H3-H4 copurified with CAF-1 from PAK2-depleted cells than control cells (Fig. 3C, cf. lane 9 and lane 10). In a reciprocal immunoprecipitation experiment, more CAF-1 and less HIRA copurified with e-H3.1 and e-H3.3, respectively, in PAK2-depleted cells than control cells (Supplemental Fig. S4F). Finally, H4S47ph was detectable on H4 copurified with HIRA, but not $\mathrm{H} 4$ copurified with CAF-1 (Fig. 3C). Together, these results indicate that H4S47ph dynamically regulates the association of $\mathrm{H} 3.1-\mathrm{H} 4$ and $\mathrm{H} 3.3-\mathrm{H} 4$ with their corresponding histone chaperones in vivo.

To determine whether H4S47ph directly impacts the association of CAF-1 and HIRA with their corresponding $(\mathrm{H} 3-\mathrm{H} 4)_{2}$ molecules, we performed in vitro immunoprecipitation experiments using recombinant $(\mathrm{H} 3.1-\mathrm{H} 4)_{2}$ and $(\mathrm{H} 3.3-\mathrm{H} 4)_{2}$ tetramers with or without $\mathrm{H} 4 \mathrm{~S} 47 \mathrm{ph}$ by PAK2 in the presence or absence of ATP. HIRA preferentially bound H3.3-H4 over H3.1H4 (Fig. 3D, cf. lanes 19-20 and lanes 15-16), whereas CAF-1 preferentially bound H3.1-H4 over H3.3-H4 (Fig. 3D, cf. lanes 7-8 and lanes 11-12), providing the first in vitro evidence supporting the idea that the distinct amino acids in H3.1 and H3.3 are important for association with their corresponding histone chaperones. More importantly, H4S47ph significantly increased the association of HIRA with $\mathrm{H} 3-\mathrm{H} 4$ and reduced the association of CAF-1 with $\mathrm{H} 3-$ $\mathrm{H} 4$ in vitro, irrespective of whether $\mathrm{H} 3$ was $\mathrm{H} 3.1$ or H3.3 (Fig. 3D, cf. lanes with ATP and those without ATP; cf. Supplemental Fig. S5). Thus, both the H3.3- and H3.1-specific residues and H4S47ph distinctly regulate the association of HIRA and CAF-1 with their corresponding (H3$\mathrm{H} 4)_{2}$ tetramers in vitro.

It has been reported that the $\mathrm{C}$ terminus of HIRA binds H4 (Lorain et al. 1998). Therefore, we tested whether the C terminus of HIRA (amino acids 738-1017) was involved

D

E
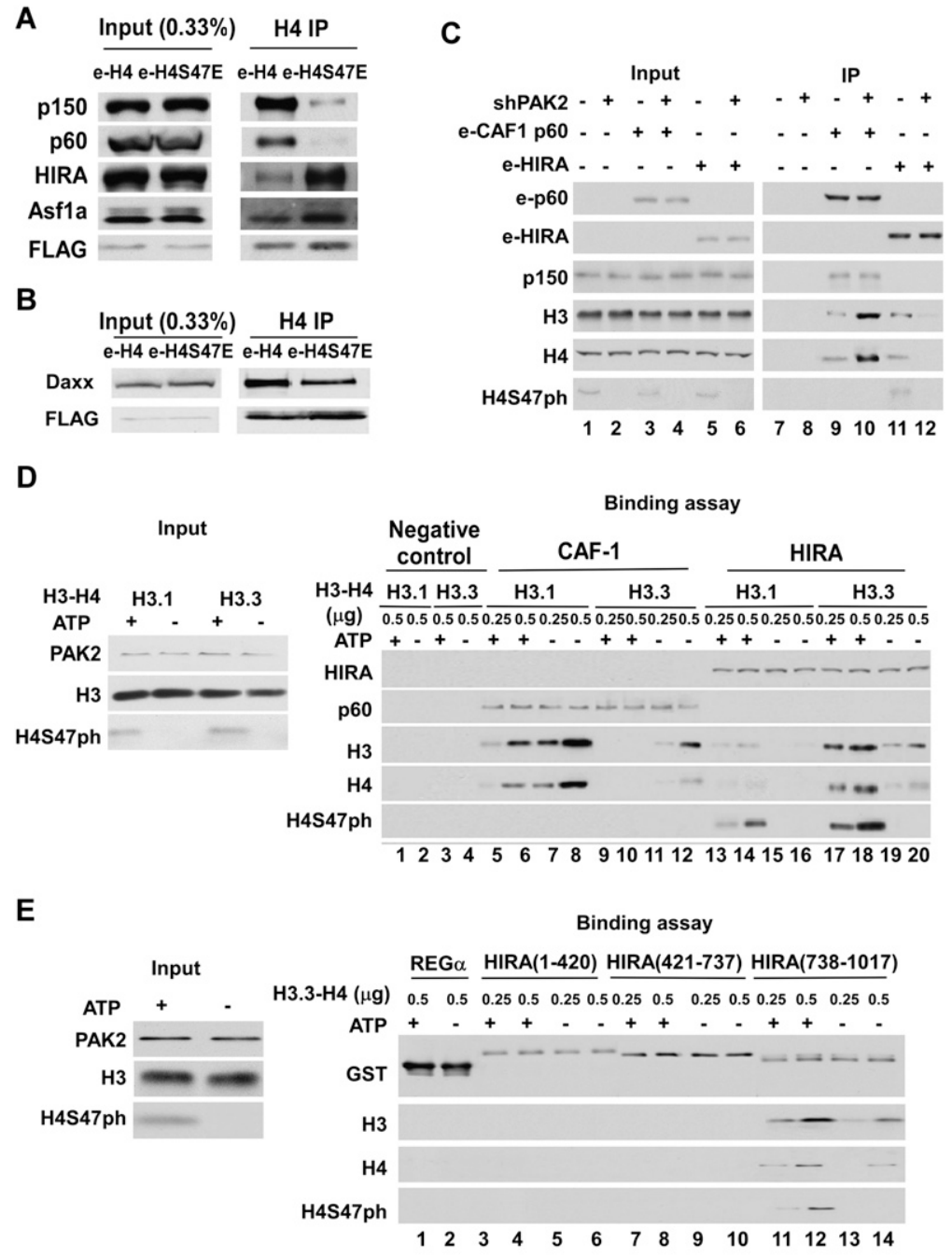

Figure 3. H4S47ph increases the interaction of HIRA and inhibits the interaction of CAF-1 with their corresponding H3-H4 molecules. $(A, B)$ Replacement of H4S47 with glutamate (H4S47E) results in a reduced binding of CAF-1 $(A)$ and Daxx $(B)$ and increased binding of HIRA $(A)$. e-H4 and e-H4S47E proteins were purified using affinity chromatography. Proteins in cell extracts (Input) and copurified proteins (IP) were detected by Western blot using the indicated antibodies. (C) Depleting PAK2 increases the binding of $\mathrm{H} 3-\mathrm{H} 4$ to CAF-1 and reduces the binding of $\mathrm{H} 3-\mathrm{H} 4$ to HIRA. CAF-1 e-p60 and e-HIRA were purified from cells infected with virus for shPAK2 $(+)$ or a nontargeting vector $(-)$ using affinity chromatography. Proteins in whole-cell extracts (Input) and copurified proteins (IP) were analyzed by Western blot using the indicated antibodies. (D) H4S47ph increases the binding of HIRA with recombinant $\mathrm{H} 3.3-\mathrm{H} 4$ and reduces the binding of CAF-1 with $\mathrm{H} 3.1-\mathrm{H} 4$ in vitro. Drosophila $(\mathrm{H} 3.1-\mathrm{H} 4)_{2}$ or $(\mathrm{H} 3.3-$ $\mathrm{H} 4)_{2}$ tetramers were purified from $E$. coli and incubated with PAK2 in the presence $(+)$ or absence of ATP (-). CAF-1 or HIRA were then mixed with two different amounts of these reaction mixtures and immunoprecipitated (IP) using antibodies against Flagtagged CAF-1 p60 and HA-tagged HIRA. Proteins were analyzed by Western blot using the indicated antibodies. (E) The C terminus of HIRA binds to $\mathrm{H} 3.3-\mathrm{H} 4 \mathrm{~S} 47 \mathrm{ph}$ preferentially. GST pull-down experiments were performed using three recombinant HIRA fragments (amino acids, 1-420, 421-737, and 738-1017) that were mixed with reaction mixtures containing $\mathrm{H} 3.3-\mathrm{H} 4$ and PAK2 with or without ATP as described in $D$, and proteins bound to different HIRA fragments were detected by Western blot.

in binding $\mathrm{H} 3.3-\mathrm{H} 4 \mathrm{~S} 47 \mathrm{ph}$ molecules. We found that the C terminus of HIRA (738-1017) bound to H3.3-H4, whereas the $\mathrm{N}$ terminus $(1-420)$ and middle region (421-737) did not. Importantly, H4S47ph increased the association of HIRA (738-1017) with H3.3-H4 (Fig. 3E). 
Thus, the C terminus of HIRA is likely involved in the recognition of H4S47ph.

\section{Alterations of H4S47ph affect the chromatin binding of CAF-1 and HIRA}

In budding yeast, the chromatin binding of CAF-1 depends, in part, on its ability to bind H3-H4 (Li et al. 2008). We therefore determined how altered levels of H4S47ph (using either the phosphatase inhibitor OA or PAK2 depletion) affect the chromatin binding of CAF-1 and HIRA. Proteins in HeLa cells were fractionated to the soluble cytosolic (S2), nucleoplasmic (S3), and chromatin-bound (P3) fractions according to a published protocol (Mendez and Stillman 2000) and were monitored by Western blot. As expected, the majority of tubulin was found in the S2 fraction compared with proteins in total cell extracts (TCEs), whereas the majority of histone $\mathrm{H} 3$ was in the $\mathrm{P} 3$ fraction, irrespective of treatment with OA or PAK2 depletion (Fig. 4A,B). Cells treated with OA exhibited a significant increase in H4S47ph in the TCE (Fig. 4A, cf. lanes 5 and 1), the S2 fraction (Fig. 4A, cf. lanes 6 and 2), and the chromatin-bound $\mathrm{P} 3$ fraction (Fig. 4A, cf. lanes 8 and 4), whereas PAK2 levels in these fractions did not change. The level of HIRA on chromatin increased, whereas the chromatin binding of CAF-1 (as revealed by both p150 and p60 subunits) was reduced in cells treated with OA (Fig. 4A, cf. lanes 8 and 4, and lanes 12-14 and 9-11). In contrast, less HIRA and more CAF-1 bound to chromatin in PAK2-depleted cells (Fig. 4B, cf. lanes 8 and 4, and lanes 12-15 and 9-11). This dynamic change in the chromatin binding of CAF-1 and HIRA following OA treatment or PAK2 depletion was confirmed by immunofluorescence after Triton X-100 extraction. The nuclear staining intensity of HIRA was increased, whereas that of CAF-1 p60 was significantly decreased, in OA-treated cells (Fig. 4C,D). The opposite effect was observed in PAK2-depleted cells (Fig. $4 \mathrm{E}, \mathrm{F})$. Neither OA treatment nor PAK2 depletion altered the overall level of HIRA and CAF-1 p60 to a significant degree as detected by Western blot (Fig. 4A,B, cf. lanes labeled TCE) or by immunofluorescence of CAF-1 p60 and HIRA without extraction (Supplemental Fig. S6). These results indicate that PAK2 and H4S47ph differentially regulate the chromatin association of CAF-1 and HIRA, providing further support for the idea that H4S47ph increases the association of HIRA with $\mathrm{H} 3.3-\mathrm{H} 4$ and decreases the association of CAF-1 with $\mathrm{H} 3.1-\mathrm{H} 4$, thereby dynamically regulating $\mathrm{H} 3.3-\mathrm{H} 4$ and H3.1-H4 nucleosome assembly.

Previous mutational studies have shown that the three distinct residues $(87,89$, and 91$)$ of $\mathrm{H} 3.3$ are important in specifying its chromatin localization and nucleosome assembly pathway (Ahmad and Henikoff 2002; Goldberg et al. 2010). We presented the following evidence supporting the idea that both $\mathrm{H} 3.3$-specific residues and $\mathrm{H} 4 \mathrm{~S} 47 \mathrm{ph}$ increase the binding affinity of HIRA with $\mathrm{H} 3.3-\mathrm{H} 4$ and thereby promote nucleosome assembly of H3.3-H4. First, we show that HIRA preferentially binds H3.3-H4 over

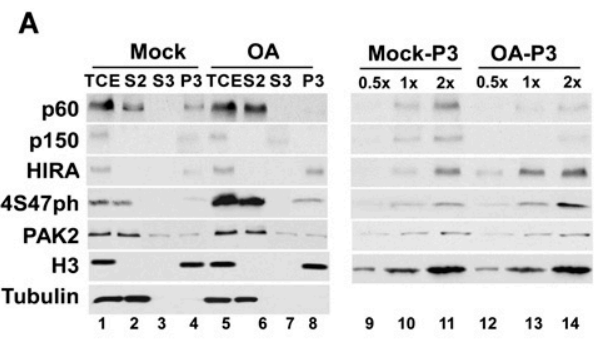

C
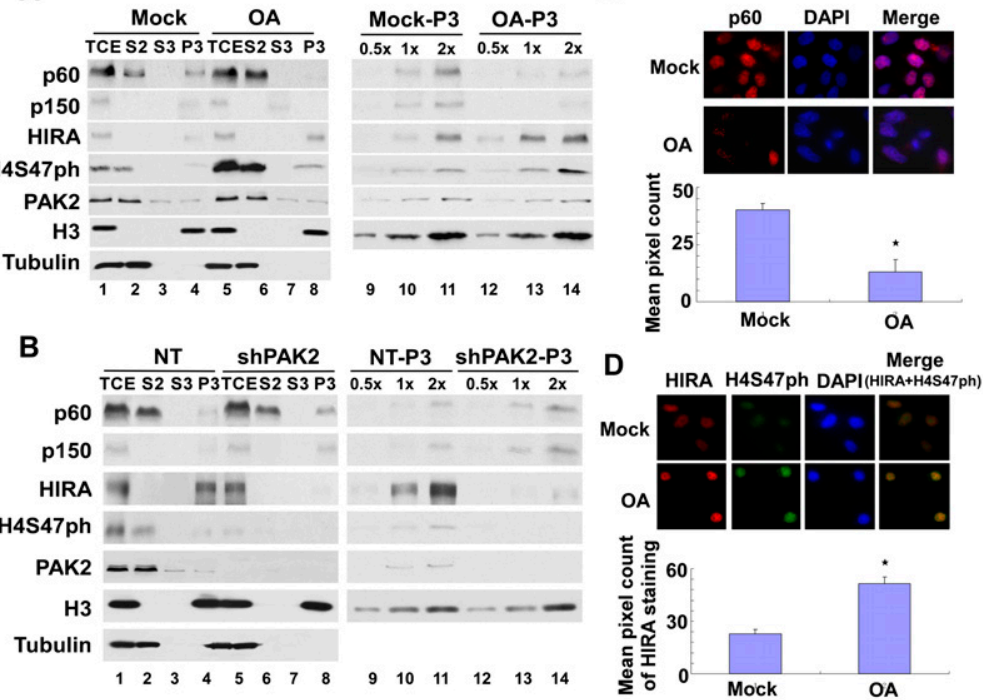

$\mathbf{E}$

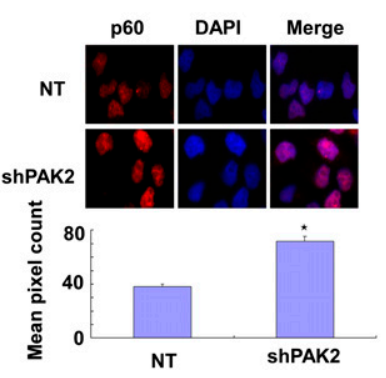

$\mathbf{F}$

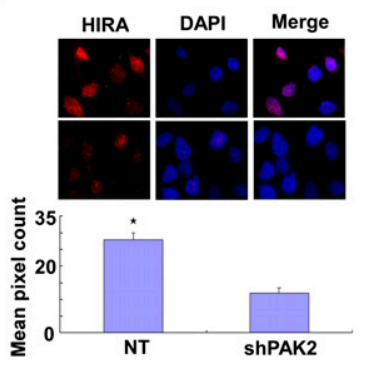

Figure 4. The chromatin binding of HIRA and CAF-1 is regulated by H4S47ph. (A) Cells treated with OA result in increased $\mathrm{H} 4 \mathrm{~S} 47 \mathrm{ph}$, increased chromatin binding of ChA, and reduced chromatin binding of CAF-1. HeLa cells were treated with OA $100 \mathrm{nM}$ ) or DMSO (Mock) for $30 \mathrm{~min}$. The S2, S3, and P3 fractions were collected. (Left panel) Proteins in TCEs and the S2, S3, and P3 fractions were analyzed by Western blot using the indicated antibodies. (Right panel) For comparison, three Depletion of PAK2 results in an increased chromatin association of CAF-1 and reduced chromatin association of HIRA. Chromatin fractionation was performed as described in $A$ except that cells with or without PAK2 depletion were used. $(C, D)$ The chromatin binding of CAF- 1 and HIRA is affected in HeLa cells treated with OA as detected by immunofluorescence. HeLa cells treated with OA or DMSO (mock) were extracted with $0.5 \%$ Triton X-100 before fixation for immunofluorescence using antibodies against p60 or HIRA. The mean pixel count of 200 nuclei was determined by Photoshop. The statistical significance of the difference between OA and mock treatment was analyzed using a $t$-test $(P<0.05) .(E, F)$ PAK2 depletion results in an increase in the chromatin binding of CAF-1 and reduced chromatin binding of HIRA. The experiments were performed as described in $C$ and $D$ except that cells with and without PAK2 depletion were used.
$\mathrm{H} 3.1-\mathrm{H} 4$ in vitro, providing an explanation for the importance of the H3.3-specific residues for specifying nucleosome assembly of H3.3. Second, H4S47ph does not differentiate $\mathrm{H} 3.1$ from $\mathrm{H} 3.3$. Instead, it increases the binding affinity of HIRA to $\mathrm{H} 3.3-\mathrm{H} 4$ and reduces the binding affinity of CAF-1 to $\mathrm{H} 3.1-\mathrm{H} 4$ in vivo. Interestingly, $\mathrm{H} 4 \mathrm{~S} 47 \mathrm{ph}$ increases the binding of $\mathrm{H} 3-\mathrm{H} 4$ to HIRA and reduces the binding affinity of CAF-1 to $\mathrm{H} 3-\mathrm{H} 4$ in vitro, irrespective of whether H3.1 or H3.3 is used (Fig. 3D). These results suggest that $\mathrm{H} 4 \mathrm{~S} 47 \mathrm{ph}$ regulates the binding of HIRA and CAF-1 with their corresponding $\mathrm{H} 3-\mathrm{H} 4$ molecules independent of the distinct residues of $\mathrm{H} 3.1$ and H3.3. Based on these results, we propose a step-wise model to explain how H4S47ph promotes HIRA-mediated 
nucleosome assembly of $\mathrm{H} 3.3-\mathrm{H} 4$ and inhibits CAF-1mediated nucleosome assembly of H3.1-H4. First, the $\mathrm{H} 3.1$ - and H3.3-specific residues facilitate recognition of H3.1-H4 and H3.3-H4 molecules by CAF-1 and HIRA, respectively. $\mathrm{H} 4 \mathrm{~S} 47 \mathrm{ph}$ then increases the binding affinity of HIRA to $\mathrm{H} 3.3-\mathrm{H} 4$, thereby promoting assembly of H3.3-H4S47ph into nucleosomes. On the other hand, H4S47ph prevents CAF-1 from binding to H3.1-H4S47ph and thus inhibits assembly of H3.1-H4S47ph into nucleosomes (Supplemental Fig. S7). H3.3 is known to be involved in gene transcription and heterochromatin formation (Elsaesser et al. 2010; Szenker et al. 2011); it would be interesting to determine whether H4S47ph and PAK2 also regulate these physiological processes.

\section{Materials and methods}

\section{Cell culture, transfection, and infection}

$293 \mathrm{~T}$ and HeLa cells were grown in DMEM medium (GIBCO) supplemented with $10 \%$ fetal calf serum and $1 \%$ penicillin/streptomycin. Stable cell lines (including those expressing e-H3.1, e-H3.3, e-H4, and e-H4S47E, each tagged with both the Flag and HA epitopes; HIRA tagged with the HA epitope; CAF-1p60 tagged with both Flag and 6-histidine; and Asf1a and Asflb tagged with both Flag and 6-histidine epitopes) were grown in the presence of $1 \mu \mathrm{g} / \mathrm{mL}$ puromycin. Cells were incubated at $37^{\circ} \mathrm{C}$ with $5 \%$ $\mathrm{CO}_{2}$. Transient transfection was performed with Lipofectamine 2000 (Invitrogen) according to the manufacturer's instructions. Lentivirus expressing PAK2 shRNAs and those expressing wild-type and mutant $\mathrm{H} 4$ proteins were packaged using $293 \mathrm{~T}$ cells and infected into targeting cells following protocols provided by Sigma-Aldrich.

\section{ChIP assay and real-time PCR}

Cells were cross-linked with $1 \%(\mathrm{v} / \mathrm{v})$ formaldehyde for $10 \mathrm{~min}$ at room temperature and quenched by the addition of $0.125 \mathrm{M}$ glycine. Cells were then resuspended in lysis buffer $(50 \mathrm{mM}$ HEPES at $\mathrm{pH} 7.5,1 \%$ Triton X-100, $140 \mathrm{mM} \mathrm{NaCl}, 1 \mathrm{mM}$ EDTA, $0.1 \%[\mathrm{w} / \mathrm{v}]$ sodium deoxycholate, protease inhibitors) and sonicated in a Bioruptor to achieve a mean DNA fragment size of 0.5 - to $1-\mathrm{kb}$ base pairs. After clarification by centrifugation, supernatants were incubated with Flag or $\mathrm{H} 4 \mathrm{~S} 47 \mathrm{ph}$ antibodies overnight at $4^{\circ} \mathrm{C}$. Following incubation, the procedures were performed as described previously (Li et al. 2008). Immunoprecipitated DNA was analyzed on a Bio-Rad real-time PCR machine. The primers used in this study are listed in Supplemental Table 1.

Other procedures-including immunofluorescence, chromatin fractionation assays, and protein purification using affinity chromatography-are described in the Supplemental Material.

\section{Acknowledgments}

We thank Drs. Peter Adams, Yoshihiro Nakatani, Alain Verreault, Genevieve Almouzni, and Scott Lowe for plasmids and antibodies used in this study. We thank Michelle Zeman and Erika Cline, two summer students, for cloning and making Asfla and Asflb stable cell lines, and Hui Zhou for purifying H3.3-H4 tetramers. We thank Qing Li and Rebecca Burgess for their critical reading of this manuscript. This work was supported by NIH grants GM72719/GM81838 to Z.Z. and GM45436/CA13016 to B.S. Z.Z. is a scholar of the Leukemia and Lymphoma Society.

\section{References}

Ahmad K, Henikoff S. 2002. The histone variant H3.3 marks active chromatin by replication-independent nucleosome assembly. Mol Cell 9: 1191-1200.

Benner GE, Dennis PB, Masaracchia RA. 1995. Activation of an S6/H4 kinase (PAK 65) from human placenta by intramolecular and intermolecular autophosphorylation. J Biol Chem 270: 21121-21128.

Bokoch GM. 2003. Biology of the p21-activated kinases. Annu Rev Biochem 72: 743-781.

Burgess RJ, Zhou H, Han J, Zhang Z. 2010. A role for Gcn5 in replicationcoupled nucleosome assembly. Mol Cell 37: 469-480.
Chen CC, Carson IJ, Feser J, Tamburini B, Zabaronick S, Linger J, Tyler JK. 2008. Acetylated lysine 56 on histone H3 drives chromatin assembly after repair and signals for the completion of repair. Cell 134: $231-243$.

Couldrey C, Carlton MB, Nolan PM, Colledge WH, Evans MJ. 1999. A retroviral gene trap insertion into the histone 3.3A gene causes partial neonatal lethality, stunted growth, neuromuscular deficits and male sub-fertility in transgenic mice. Hum Mol Genet 8: 2489-2495.

Das C, Lucia MS, Hansen KC, Tyler JK. 2009. CBP/p300-mediated acetylation of histone $\mathrm{H} 3$ on lysine 56. Nature 459: 113-117.

Drane P, Ouararhni K, Depaux A, Shuaib M, Hamiche A. 2010. The deathassociated protein DAXX is a novel histone chaperone involved in the replication-independent deposition of H3.3. Genes Dev 24: 1253-1265.

Elsaesser SJ, Goldberg AD, Allis CD. 2010. New functions for an old variant: no substitute for histone H3.3. Curr Opin Genet Dev 20: 1-8.

Goldberg AD, Banaszynski LA, Noh KM, Lewis PW, Elsaesser SI, Stadler S, Dewell S, Law M, Guo X, Li X, et al. 2010. Distinct factors control histone variant $\mathrm{H} 3.3$ localization at specific genomic regions. Cell 140: 678-691.

Groth A, Rocha W, Verreault A, Almouzni G. 2007. Chromatin challenges during DNA replication and repair. Cell 128: 721-733.

Huang Z, Traugh JA, Bishop JM. 2004. Negative control of the Myc protein by the stress-responsive kinase Pak2. Mol Cell Biol 24: 1582-1594.

Jasencakova Z, Scharf AN, Ask K, Corpet A, Imhof A, Almouzni G, Groth A. 2010. Replication stress interferes with histone recycling and predeposition marking of new histones. Mol Cell 37: 736-743.

Jin C, Zang C, Wei G, Cui K, Peng W, Zhao K, Felsenfeld G. 2009. H3.3/ H2A.Z double variant-containing nucleosomes mark 'nucleosome-free regions' of active promoters and other regulatory regions. Nat Genet 41: 941-945.

Li Q, Zhou H, Wurtele H, Davies B, Horazdovsky B, Verreault A, Zhang Z. 2008. Acetylation of histone H3 lysine 56 regulates replicationcoupled nucleosome assembly. Cell 134: 244-255.

Lorain S, Quivy JP, Monier-Gavelle F, Scamps C, Lecluse Y, Almouzni G, Lipinski M. 1998. Core histones and HIRIP3, a novel histone-binding protein, directly interact with WD repeat protein HIRA. Mol Cell Biol 18: $5546-5556$.

Mendez J, Stillman B. 2000. Chromatin association of human origin recognition complex, cdc6, and minichromosome maintenance proteins during the cell cycle: assembly of prereplication complexes in late mitosis. Mol Cell Biol 20: 8602-8612.

Mito Y, Henikoff JG, Henikoff S. 2005. Genome-scale profiling of histone H3.3 replacement patterns. Nat Genet 37: 1090-1097.

Morrison AJ, Shen X. 2009. Chromatin remodelling beyond transcription: the INO80 and SWR1 complexes. Nat Rev Mol Cell Biol 10: 373-384.

Ransom M, Dennehey B, Tyler JK. 2010. Chaperoning histones during repair and replication. Cell 140: 183-195.

Ray-Gallet D, Quivy JP, Scamps C, Martini EM, Lipinski M, Almouzni G. 2002. HIRA is critical for a nucleosome assembly pathway independent of DNA synthesis. Mol Cell 9: 1091-1100.

Sakai A, Schwartz BE, Goldstein S, Ahmad K. 2009. Transcriptional and developmental functions of the H3.3 histone variant in Drosophila. Curr Biol 19: 1816-1820.

Santenard A, Ziegler-Birling C, Koch M, Tora L, Bannister AJ, Torres-Padilla ME. 2010. Heterochromatin formation in the mouse embryo requires critical residues of the histone variant H3.3. Nat Cell Biol 12: 853-862.

Schwartz BE, Ahmad K. 2005. Transcriptional activation triggers deposition and removal of the histone variant H3.3. Genes Dev 19: 804-814.

Smith S, Stillman B. 1989. Purification and characterization of CAF-I, a human cell factor required for chromatin assembly during DNA replication in vitro. Cell 58: 15-25.

Szenker E, Ray-Gallet D, Almouzni G. 2011. The double face of the histone variant H3.3. Cell Res 21: 421-434.

Tagami H, Ray-Gallet D, Almouzni G, Nakatani Y. 2004. Histone H3.1 and $\mathrm{H} 3.3$ complexes mediate nucleosome assembly pathways dependent or independent of DNA synthesis. Cell 116: 51-61. 


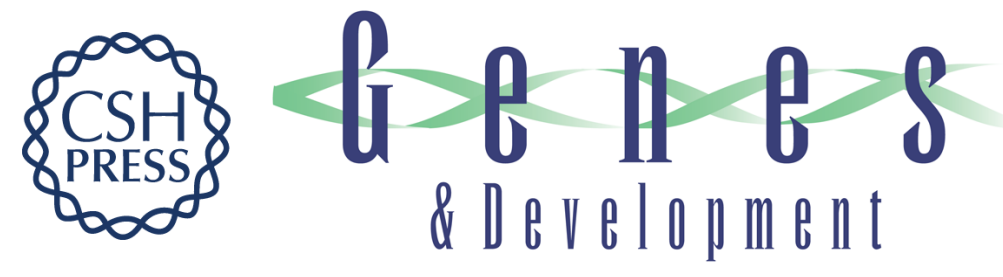

\section{Phosphorylation of H4 Ser 47 promotes HIRA-mediated nucleosome assembly}

Bin Kang, Mintie Pu, Gangqing Hu, et al.

Genes Dev. 2011, 25:

Access the most recent version at doi:10.1101/gad.2055511

\section{Supplemental http://genesdev.cshlp.org/content/suppl/2011/06/30/25.13.1359.DC1 Material}

References This article cites 27 articles, 6 of which can be accessed free at: http://genesdev.cshlp.org/content/25/13/1359.full.html\#ref-list-1

\section{License}

Email Alerting

Service

Receive free email alerts when new articles cite this article - sign up in the box at the top right corner of the article or click here.

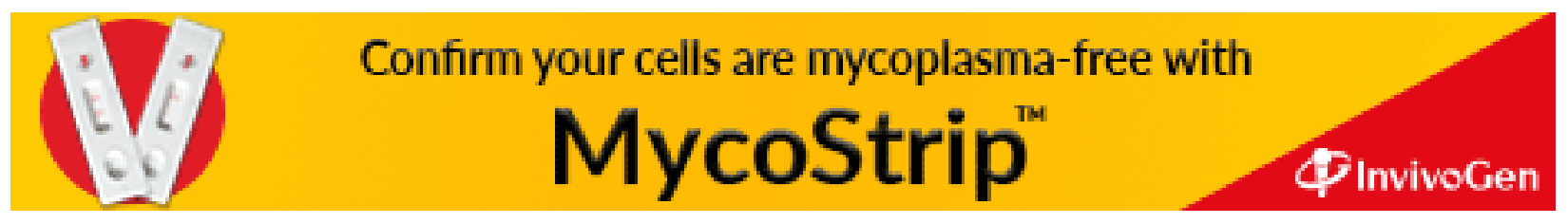

